\title{
Development of New-Onset Chronic Inflammatory Demyelinating Polyneuropathy Following Exposure to a Water-Damaged Home With High Airborne Mold Levels: A Report of Two Cases and a Review of the Literature
}

\author{
Allan Lieberman ${ }^{\mathrm{a}}$, Luke Curtis ${ }^{\mathrm{a}, \mathrm{c}}$, Andrew Campbell ${ }^{\mathrm{b}}$
}

\begin{abstract}
The exact pathological processes and triggers for chronic inflammatory demyelinating polyneuropathy (CIDP) are not well understood. We report two patients who developed CIDP after living in a badly water-damaged home which contained very high levels of airborne mold. We also present a short review of the related literature linking exposure to mold and mycotoxins with neurological problems. The patients had nerve conduction velocities, clinical exam findings, and (in one patient) a sural nerve biopsy consistent with CIDP. The patients also developed other new-onset chronic health problems including chronic fatigue, asthma, and neurocognitive problems such as memory and attention deficits. Clinicians who treat patients with CIDP and other peripheral neuropathies need to consider and document environmental and occupational exposures (such as indoor water damage and heavy mold growth) as potential triggers for neurological damage.
\end{abstract}

Keywords: Autoimmunity; Chronic inflammatory demyelinating polyneuropathy; Fungi; Molds; Mycotoxins; Water damage

\section{Introduction}

Chronic inflammatory demyelinating polyneuropathy (CIDP) is a type of acquired immune-mediated inflammatory condition affecting the peripheral nervous system. CIDP is a group of polyneuropathies which present chronically over a period of more than 8 weeks $[1,2]$. The classic form of CIDP presents with generally symmetrical proximal limb weakness, areflexia, large fiber sensory loss, numbness, tingling, gait imbalance,

Manuscript accepted for publication March 27, 2017

${ }^{a}$ Center for Occupational and Environmental Health, 7510 Northforest Drive, North Charleston, SC 29420, USA

' $T h e$ Wellness Center, 23144 Emerson Way, Land O'Lakes, FL 34639, USA ${ }^{\mathrm{c} C}$ Corresponding Author: Luke Curtis, Center for Occupational and Environmental Health, 7510 Northforest Drive, North Charleston, SC 29420, USA. Email: lcurtis@coem.com

doi: https://doi.org/10.14740/jnr413e and sometimes painful paresthesias [2, 3]. Neuropsychiatric problems such as depression, anxiety, memory and concentration difficulties, chronic fatigue, chronic pain, and sleep problems have also been commonly reported $[4,5]$. Several variants of classic CIPD are known and exact diagnosis is often difficult $[3,6,7]$, but is generally diagnosed on the basis of medical history, physical examination, and peripheral nerve studies [8].

Other characteristic findings, such as the presence of demyelination in nerve biopsies or elevated cerebral spinal protein levels, can be helpful in making the diagnosis [8]. Various studies have estimated the prevalence of CIDP to be $0.8-8.9$ per 100,000 population, with an annual incidence of $0.2-1.6$ per 100,000 [9]. The causes and triggers for CIDP development are unclear [10].

The concurrent diagnosis of two cases of CIDP in the same home led us to consider possible environmental triggers. They were exposed to documented heavy water damage and mold contamination, which were temporally related to CIDP onset. When we learned that the Louisiana Medical Center where the two were diagnosed was seeing an average of five new cases of CIDP per week in the months following Hurricane Katrina [11], the significance of an environmental trigger in a disease of unknown origin became important. The relatively low incidence of CIDP suggests that the simultaneous development of two cases of CIDP in the same home is unlikely due to chance alone. Case studies in occupational and environmental medicine are often a useful step in determining etiology of an enigmatic disorder.

\section{Case Report}

The two cases involved two brothers (AR and RR) who were 58 and 59 years old when their home flooded in 2002. AR had been generally healthy until age 58 and was running 20 miles per week. In 2002, he stayed at his brother's house 4 - 5 days per week while his condo was being remodeled. The roof had many water leaks, including 1 week in June 2002 in which a large amount of rainwater entered the house while the roof was undergoing repair for hailstone damage. The home became saturated with water during this flooding. The house was contaminated with high airborne concentrations of molds including Aspergillus/Penicillium and Chaetomium (Table 1). After 
Table 1. Fungal Spores per Cubic Meter of Air in Louisiana Home on November 26, 2005.

\begin{tabular}{lllll}
\hline & Dining room & Second bedroom countertop & Master bedroom & Outdoors near house \\
\hline Aspergillus/Penicillium & 48,086 & 116,571 & 255,000 & 0 \\
Cladosporium & 0 & 0 & 0 & 1,078 \\
Chaetomium & 18,943 & 43,714 & 11,657 & 0 \\
Others & 1,314 & 658 & 0 & 293 \\
Total & 68,343 & 160,943 & 267,657 & 1,371 \\
\hline
\end{tabular}

a month of exposure in the water- and mold-damaged house, they relocated to a hotel.

During the summer of 2002, AR experienced tingling and numbness of the feet and calves and discomfort in the right thigh and back and inability to stand on heels and toes. AR had an EMG, which indicated an acquired multi-focal sensory-motor neuropathy with demyelinating features as seen in CIDP. He had a number of abnormal nerve conduction values including an abnormally low left peroneal motor nerve velocity of 28 $\mathrm{m} / \mathrm{s}$ (normal $>44 \mathrm{~m} / \mathrm{s}$ ), an abnormally high left peroneal motor nerve latency of $85 \mathrm{~ms}$ (normal $<50 \mathrm{~ms}$ ), and an abnormally low velocity of $29 \mathrm{~m} / \mathrm{s}$ (normal $\geq 40 \mathrm{~m} / \mathrm{s}$ ) and an amplitude of $2.1 \mathrm{mV}$ (normal $>6 \mathrm{mV}$ ) for the left tibial motor nerve. He was formally diagnosed with CIDP in June 2003 after a sural nerve biopsy was consistent with CIDP. In June 2003, the patient began treatment with intravenous immunoglobulin (IVIG) which was associated with a significant improvement in his CIDP symptoms. He continues to the present with IVIG treatment or he regresses.

To rule out a possible genetic cause, as the patients were brothers, AR had genetic testing at Athena Labs (Marlborough, MA) by PCR and Sanger DNA sequencing which indicated that the patient did not have four gene mutations characteristic of Charcot-Marie-Tooth disease, which was considered in the differential diagnosis. In 2013, AR presented to one of the authors (AL) with chronic fatigue and continued nerve problems, including absence of deep tendon reflexes in all four extremities, absent vibratory sensation in lower legs, diminished touch sensation in legs, and marked atrophy of muscles in both legs.

In the 2 months following the flooding, RR, the brother of AR who lived in the same house during the June 2002 flooding incident, developed fatigue, chronic sinusitis, myalgia, brain fog and then asthma. By October 2002, RR was diagnosed with asthma and allergic fungal sinusitis. On November 11, 2002, he experienced severe total body pruritus which was treated with antihistamines and topicals with little effect. By March 2003, RR was experiencing tingling, weakness and numbness of all four extremities and some neurocognitive loss. By May 2003, RR's pruritus resolved without medication.

RR had nerve conduction studies and an EMG on June 29, 2005, diagnostic of an acquired chronic demyelinating sensorimotor polyneuropathy consistent with CIDP. Motor nerve conduction showed reduced amplitude in the left tibial $(0.8$ $\mathrm{mV})$, peroneal $(2.8 \mathrm{mV})$, and ulnar nerves $(4.2 \mathrm{mV})$. F-wave latencies were prolonged in both legs. He received treatment with IVIG starting in 2003, which helped improve his CIDP symptoms. By 2016, he still had CIDP symptoms, chronic fatigue, episodic asthma, difficulties with memory and concen- tration, and had developed new-onset moderate kidney failure (GFR of $37 \mathrm{~mL} / \mathrm{min}$ ). Kidney failure was consistent with IVIG osmotic damage reported in patients receiving long-term IVIG therapy with sucrose $[12,13]$.

\section{Environmental sampling of the home}

An environmental survey on November 20, 2002 of the Louisiana home reported that the walls of the home had been "almost entirely saturated with water". Visible mold was present throughout the house, and air and lift samples revealed multiple molds including Cladosporium, Aspergillus, and Penicillium. Water and moisture problems continued in the home for years. An environmental study of the home on November 26, 2005 reported very high total mold spore levels in the Louisiana home (Table 1).

Compared to the outdoor samples, the indoor spore per $\mathrm{m}^{3}$ of air were 50 - 195 times higher in the Louisiana home. By comparison, a review of 85 worldwide residential buildings reported average total spore concentrations of 913 spores $/ \mathrm{m}^{3}$ of air, with a range of $68-2,384$ spores $/ \mathrm{m}^{3}$ [14]. Levels of Chaetomium were very high in the home. One study of 9,619 indoor air viable samples reported that Chaetomium was found in only $3 \%$ of the samples with a mean concentration of 0.36 viable spores $/ \mathrm{m}^{3}$ of air [15]. The high Chaetomium levels are an indicator of long-term water damage as Chaetomium generally grows indoors only in water-damaged buildings and is not readily airborne [16].

\section{Discussion}

This case series documents that indoor residential exposure to heavy water damage and high levels of indoor molds was associated with the development of CIDP. The relative rarity of CIDP (annual incidence of 0.2 - 1.6 per 100,000 year) [9] suggests that the simultaneous development of two cases of CIDP at the same time in the same household is unlikely due to chance alone.

The triggers for CIDP development and pathogenesis are unclear [10]. Several case reports have associated the development of CIDP with vaccination or HIV infection [17, 18]. Epidemiological studies have reported that CIDP is often associated with other conditions such as monoclonal gammopathy of undetermined significance (MGUS), autoimmune disorders (lupus, rheumatoid arthritis, Crohn's disease, and multiple sclerosis), diabetes, renal failure, and some forms of cancer 
[2]. The pathogenesis of CIDP is believed to be related to autoimmune processes involving both the cellular and humoral systems including antibodies to the myelin sheath antigens $[2$, $3,8,10,19,20]$.

Molds produce a wide range of allergens and secondary metabolites known as mycotoxins [21-23]. Exposure to heavy indoor mold growth has been associated with many types of central and peripheral nerve problems including headaches, postural imbalance, concentration and memory problems, tremors, and distal numbness weakness [22-26]. A study of 119 patients with heavy indoor mold exposure and symptoms of peripheral neuropathy reported that $99(83 \%)$ had significantly impaired function in one or more of four motor and three sensory nerves as compared to only one of $20(5 \%)$ of controls [26]. Fifty-five of these 119 mold exposed patients (46\%) were diagnosed with CIDP by a board certified neurologist [27]. Another study reported significantly higher levels of auto-antibodies to central nervous system (CNS) myelin, peripheral nervous system (PNS) myelin, nuclear membranes, and smooth muscle in a group of 209 patients heavily exposed to molds in water-damaged buildings as compared to 28 healthy controls [24]. A neuropsychiatric study reported that 105 mold exposed patients had significantly worse reaction times, memory, concentration, balance and manual dexterity as compared to 202 controls, suggesting that molds can cause a wide range of deficits to both the CNS and PNS [25].

Animal studies suggest that some molds and mycotoxins may be toxic to myelin nerve sheaths. Demyelinating disorders have been reported in rats orally exposed to the mycotoxin fumonisin B1 (produced by Fusarium) [28] and in monkeys and cattle exposed to diplodiatoxin (produced by Diplodia maydis) tainted feeds $[29,30]$. Other studies with lab animals and cultured human nerve cells suggest that low level exposure to the mycotoxin satratoxin (produced by Stachybotrys fungi) is associated with nerve cell inflammation, oxidative damage, damage to the blood brain barrier, and excessive nerve cell death [31-33].

Exposure to molds, mycotoxins, and other water damagerelated bioaerosols (such as bacteria and their toxins) may increase the risk of CIDP by stimulating auto-immune responses. Various studies have reported that auto-antibodies to proteins and glycoproteins associated with the myelin sheath are commonly seen in both mold exposed patients [24] and in patients with CIDP and related disorders [34]. Exposure to bacterial and viral infections may play a role in increased risk of autoimmune disease $[35,36]$. Many types of environmental exposures such as smoking, pesticides, organic solvents, silica and other particulates, and heavy metals like lead and mercury, have been linked to a wide range of auto-immune diseases like rheumatoid arthritis, lupus, type 1 diabetes, Sjogren's disease, and autoimmune thyroid disease [35, 37-40].

\section{Conclusions}

In summary, our case series reports two cases of CIDP developing after long-term exposure to a water-damaged home with very high airborne mold levels. Clinicians who treat patients with CIDP and other peripheral neuropathies need to consid- er and document environmental and occupational exposures (such as indoor water intrusion and mold) as potential triggers for neurological damage.

\section{Funding Support}

This case series received no outside funding.

\section{Abbreviations}

CIDP: chronic inflammatory demyelinating polyneuropathy; CNS: central nervous system; PNS: peripheral nervous system

\section{References}

1. Dimachkie MM, Saperstein DS. Acquired immune demyelinating neuropathies. Continuum (Minneap Minn). 2014;20(5 Peripheral Nervous System Disorders):1241-1260.

2. Gorson KC, Katz J. Chronic inflammatory demyelinating polyneuropathy. Neurol Clin. 2013;31(2):511-532.

3. Dimachkie MM, Barohn RJ. Chronic inflammatory demyelinating polyneuropathy. Curr Treat Options Neurol. 2013;15(3):350-366

4. Rajabally YA, Seri S, Cavanna AE. Neuropsychiatric manifestations in inflammatory neuropathies: A systematic review. Muscle Nerve. 2016;54(1):1-8.

5. Merkies IS, Kieseier BC. Fatigue, Pain, Anxiety and Depression in Guillain-Barre Syndrome and Chronic Inflammatory Demyelinating Polyradiculoneuropathy. Eur Neurol. 2016;75(3-4):199-206.

6. Van den Bergh PY, Hadden RD, Bouche P, Cornblath DR, Hahn A, Illa I, Koski CL, et al. European Federation of Neurological Societies/Peripheral Nerve Society guideline on management of chronic inflammatory demyelinating polyradiculoneuropathy: report of a joint task force of the European Federation of Neurological Societies and the Peripheral Nerve Society - first revision. Eur J Neurol. 2010;17(3):356-363.

7. Cornblath DR, Gorson KC, Hughes RA, Merkies IS. Observations on chronic inflammatory demyelinating polyneuropathy: A plea for a rigorous approach to diagnosis and treatment. J Neurol Sci. 2013;330(1-2):2-3.

8. Dalakas MC. Advances in the diagnosis, pathogenesis and treatment of CIDP. Nat Rev Neurol. 2011;7(9):507517.

9. Mahdi-Rogers M, Hughes RA. Epidemiology of chronic inflammatory neuropathies in southeast England. Eur J Neurol. 2014;21(1):28-33.

10. Svahn J, Antoine JC, Camdessanche JP. Pathophysiology and biomarkers in chronic inflammatory demyelinating polyradiculoneuropathies. Rev Neurol (Paris). 2014;170(12):808-817.

11. Willeford A. Personal Communication with Jim Williford. An average of 5 new cases of CIDP per week were 
seen at the Louisiana State University in the months following Katrina and its associated heavy flooding. 2016.

12. Shah S, Vervan M. Use of i.v. immune globulin and occurrence of associated acute renal failure and thrombosis. Am J Health Syst Pharm. 2005;62(7):720-725.

13. Dantal J. Intravenous immunoglobulins: in-depth review of excipients and acute kidney injury risk. Am J Nephrol. 2013;38(4):275-284.

14. Gots RE, Layton NJ, Pirages SW. Indoor health: background levels of fungi. AIHA J (Fairfax, Va). 2003;64(4):427-438.

15. Shelton BG, Kirkland KH, Flanders WD, Morris GK. Profiles of airborne fungi in buildings and outdoor environments in the United States. Appl Environ Microbiol. 2002;68(4):1743-1753.

16. Samson RA, Houbraken J, Thrane U, Frisvad JC, Andersen B. Food and Indoor Fungi. Utecht, The Netherlands: CBS- KNAW Fungal Biodversity Centre, 2010.

17. Gable KL, Afshari Z, Sufit RL, Allen JA. Distal acquired demyelinating symmetric neuropathy after vaccination. J Clin Neuromuscul Dis. 2013;14(3):117-122.

18. Rajabally Y, Vital A, Ferrer X, Vital C, Julien J, Latour $\mathrm{P}$, Vandenberghe A, et al. Chronic inflammatory demyelinating polyneuropathy caused by HIV infection in a patient with asymptomatic CMT 1A. J Peripher Nerv Syst. 2000;5(3):158-162.

19. Kieseier BC, Dalakas MC, Hartung HP. Immune mechanisms in chronic inflammatory demyelinating neuropathy. Neurology. 2002;59(12 Suppl 6):S7-12.

20. Toyka KV, Gold R. The pathogenesis of CIDP: rationale for treatment with immunomodulatory agents. Neurology. 2003;60(8 Suppl 3):S2-7.

21. Brase S, Encinas A, Keck J, Nising CF. Chemistry and biology of mycotoxins and related fungal metabolites. Chem Rev. 2009;109(9):3903-3990.

22. Curtis L, Lieberman A, Rea W, Stark M, Vetter M. Advere human health effects of indoor molds. J Nutr Environ Med. 2004;14:261-274.

23. Hope J. A review of the mechanism of injury and treatment approaches for illness resulting from exposure to water-damaged buildings, mold, and mycotoxins. ScientificWorldJournal. 2013;2013:767482.

24. Gray MR, Thrasher JD, Crago R, Madison RA, Arnold L, Campbell AW, Vojdani A. Mixed mold mycotoxicosis: immunological changes in humans following exposure in water-damaged buildings. Arch Environ Health. 2003;58(7):410-420.

25. Kilburn KH. Neurobehavioral and pulmonary impairment in 105 adults with indoor exposure to molds compared to 100 exposed to chemicals. Toxicol Ind Health. 2009;25(9-10):681-692.

26. Campbell AW, Thrasher JD, Madison RA, Vojdani A, Gray MR, Johnson A. Neural autoantibodies and neurophysiologic abnormalities in patients exposed to molds in water-damaged buildings. Arch Environ Health.
2003;58(8):464-474.

27. Campbell AW. Personal Communication. Number of patients formally diagnosed with chronic inflammatory demyelinating polyneuropathy out of a group of 119 mold exposed patients in a 2003 study. 2016.

28. Carratu MR, Cassano T, Coluccia A, Borracci P, Cuomo V. Antinutritional effects of fumonisin B1 and pathophysiological consequences. Toxicol Lett. 2003;140-141:459463.

29. Odriozola E, Odeon A, Canton G, Clemente G, Escande A. Diplodia maydis: a cause of death of cattle in Argentina. N Z Vet J. 2005;53(2):160-161.

30. Fincham JE, Hewlett R, de Graaf AS, Taljaard JJ, Steytler JG, Rabie CJ, Seier JV, et al. Mycotoxic peripheral myelinopathy, myopathy, and hepatitis caused by Diplodia maydis in vervet monkeys. J Med Primatol. 1991;20(5):240-250.

31. Carey SA, Plopper CG, Hyde DM, Islam Z, Pestka JJ, Harkema JR. Satratoxin-G from the black mold Stachybotrys chartarum induces rhinitis and apoptosis of olfactory sensory neurons in the nasal airways of rhesus monkeys. Toxicol Pathol. 2012;40(6):887-898.

32. Islam Z, Harkema JR, Pestka JJ. Satratoxin G from the black mold Stachybotrys chartarum evokes olfactory sensory neuron loss and inflammation in the murine nose and brain. Environ Health Perspect. 2006;114(7):1099-1107.

33. Karunasena E, Larranaga MD, Simoni JS, Douglas DR, Straus DC. Building-associated neurological damage modeled in human cells: a mechanism of neurotoxic effects by exposure to mycotoxins in the indoor environment. Mycopathologia. 2010;170(6):377-390.

34. Nobile-Orazio E, Giannotta C. Testing for anti-glycolipid IgM antibodies in chronic immune-mediated demyelinating neuropathies. J Peripher Nerv Syst. 2011;16(Suppl 1):18-23.

35. Wang L, Wang FS, Gershwin ME. Human autoimmune diseases: a comprehensive update. J Intern Med. 2015;278(4):369-395.

36. Bogdanos DP, Smyk DS, Rigopoulou EI, Sakkas LI, Shoenfeld Y. Infectomics and autoinfectomics: a tool to study infectious-induced autoimmunity. Lupus. 2015;24(4-5):364-373.

37. Maciejewska A. Health effects of occupational exposure to crystalline silica in the light of current research results. Med Pr. 2014;65(6):799-818.

38. Barragan-Martinez C, Speck-Hernandez CA, MontoyaOrtiz G, Mantilla RD, Anaya JM, Rojas-Villarraga A. Organic solvents as risk factor for autoimmune diseases: a systematic review and meta-analysis. PLoS One. 2012;7(12):e51506.

39. Bodin J, Stene LC, Nygaard UC. Can exposure to environmental chemicals increase the risk of diabetes type 1 development? Biomed Res Int. 2015;2015:208947.

40. Duntas LH. Environmental factors and thyroid autoimmunity. Ann Endocrinol (Paris). 2011;72(2):108-113. 